\section{PRELIMINARY INSIGHTS INTO THE IMPACT OF TUMOR MICROBIOME IN HEAD AND NECK SQUAMOUS CELL CARCINOMA}

${ }^{1}$ Vidhya Karivedu*, ${ }^{2}$ Rebecca Hoyd, ${ }^{3}$ Caroline Wheeler, ${ }^{1}$ Sachin Jhawar, ${ }^{1}$ Priyanka Bhateja, ${ }^{1}$ Marcelo Bonomi, ${ }^{3}$ Daniel Spakowicz. ${ }^{1}$ The Ohio State University Wexner Medical Center, Columbus, OH, USA; ${ }^{2}$ The Ohio State University College of Medicine, Columbus, OH, USA

${ }^{3}$ The Ohio State University, Columbus, OH, USA

Background Head and neck squamous cell carcinoma (HNSCC) is a heterogeneous set of distinct malignancies. Recognized prognostic factors rely on clinical and biological features, consisting mainly of stage, site of disease, performance status, comorbidities, smoking history and human papilloma virus (HPV) status. However, patients clustered by these parameters still differ in their clinical behavior and therapy response. The impact of the tumor microbiome on human disease has been explored and discussed extensively. Evaluating the tumor microbiome is a promising new approach that could be used as a prognostic and predictive tool in HNSCC, with the potential for improved treatment options and better clinical outcomes.

Methods We utilized The Cancer Genome Atlas (TCGA) database to obtain RNA sequencing (RNAseq) data to identify microbes in HNSCC samples. We utilized ExoTIC, "Exogenous sequences in Tumors and Immune cells," a tool recently developed by Spakowicz et al. ExoTIC takes raw RNAseq reads and carefully aligns them to both human and nonhuman reference genomes to identify low-abundance microbes. We performed Cox proportional hazards regression to identify the microbes associated with overall survival (OS), controlling for age, stage, and smoking status.

Results We evaluated 498 RNAseq samples from TCGA (table 1). ExoTIC identified 5838 microbes including bacteria, viruses and fungi, of which 330 were statistically associated with OS. Interestingly, 20\% $(\mathrm{n}=100)$ of samples had HPV virus which was significantly associated with improved OS (HR 0.59, CI $0.4-0.9, \mathrm{p}<0.01$ ). There were also several other viruses and bacteria associated with significantly improved OS.

Abstract 837 Table 1 Patient characteristics of TCGA dataset

\begin{tabular}{|l|l|}
\hline \multicolumn{2}{|c|}{ Overall } \\
\hline$n$ & 498 \\
\hline Age (mean (SD)) & $61.08(11.92)$ \\
\hline Sex = male (\%) & $366(73.3)$ \\
\hline Cancer Stage (\%) & $19(3.9)$ \\
\hline Stage I & $94(19.4)$ \\
\hline Stage II & $102(21)$ \\
\hline Stage III & $270(55.7)$ \\
\hline Stage IV & $108(21.6)$ \\
\hline Primary Site (\%) & $74(15)$ \\
\hline Oral cavity & $9(1.8)$ \\
\hline Oropharynx & $111(22.2)$ \\
\hline Hypopharynx & $196(39.3)$ \\
\hline Larynx & $218(43.7)$ \\
\hline Other &
\end{tabular}

Conclusions We found the presence of certain microbes in tumor biopsies statistically correlated with OS in HNSCC patients. This supports further study into the presence and correlation of specific microbes with tumor subsite and outcomes. Assessing individual characteristics of a HNSCC subtype with its particular microenvironment (e.g., microbes) can lead to personalized treatment insights and improved outcomes. Our future research will validate and correlate the microbial profile of HNSCC subtypes with clinical outcomes retrospectively and prospectively.

http://dx.doi.org/10.1136/jitc-2021-SITC2021.837 